\title{
Survey Paper on School Bus Environment Monitoring by Advance GSM and DGPS Technology
}

\author{
Ayodhya R. Gutte ${ }^{1}$, H. K. Bhangale ${ }^{2}$ \\ ${ }^{1}$ PG Student, M.E. Digital Electronics, G H Raisoni Institute of engineering and Management, Jalgaon, India \\ ${ }^{2}$ Professor and Head of the Department, Department of Electronics Engineering, G H Raisoni institute of engineering and mangement, \\ Jalgaon, India
}

\begin{abstract}
In this paper survey is done on paper monitoring and tracking of school bus by advance GSM and GPS technology implemented on RTOS and embedded board. It is proposed for continuously monitoring a school bus or any vehicle for safety of traveler's travelling with the use of available technologies at that time. A vehicle tracking system at real time consist of electronic device placed inside of vehicle. Continuously monitoring can be done by internet or by special software.
\end{abstract}

Keywords: GLONASS, GNSS, GPS, GSM, IT

\section{Introduction}

Now a days safety of traveler is most important thing. With more vehicles on roads now than ever before, vehicle tracking has become an incredibly important tool for everyone who wish to evade such problematic and time consuming traffic. To avoid these types of problem vehicle tracking system provide us real time location of vehicle. GPS and GSM technology helps us mapping the real time location and reporting all this information to the monitoring side. Therefore comparatively we can monitor our vehicle. By using GPS we get to know about where our vehicle is, where it has been, how far it is from our location. Use of tracking technology is most important in today's world. Since it has been in use from many years ago. There were other technologies for tracking of vehicle or location invented by another countries.

\section{Global Navigation satellite system}

\section{A. GPS}

The Global Positioning System is a satellite radio navigation system developed by the Department of Defense (DoD) owned by the United States Government (USG) and operated by the United States Air Force.

GPS provide positioning, navigation, and timing services to military and civilian users through worldwide basis since it was first launched in 1978. An unlimited number of users with a civil or military GPS receiver can measure accurate time and location, in any condition of weather, day or night, anywhere in the world [3].

The system makes use of a earth orbit satellite constellation which transmits microwave signals allows a GPS receiver to determine its velocity, location and time etc. Different types of positioning can be carried out using GPS receivers depending on the type of measurements and correction values used in the navigation solution.
A GPS receiver can measure the apparent range between satellite and receiver (pseudo range), [4]using the code phase measurements, which provides the instantaneous ranges to the satellites, or the carrier phase measurements i.e. is the difference between the phase of the carrier signal present at the receiver and the carrier received from a satellite at the instant of the measurement.

The phase carrier measurement is given in a fraction of a cycle, but this does not contain none of information about the integer ambiguity i.e. number of cycles complete. Requirements for developing the GPS systems from the signaling point of view are as follows:

a) For avoiding interference from other satellites, multiple access capabilities should be developed

b) Avoiding some amount of multipath interference.

c) Minimization of interference from jamming of signal etc. up to a certain level.

d) Low power signal so that it should not interfere with the microwave line

Features:

- Satellite Constellation: 32 Satellites

- Multiple Access: CDMA

- Modulation schemes used: BPSK

- Center Frequency: 1575.42 MHz, 1227.60 MHz, 1176.45 $\mathrm{MHz}$

\section{B. GLONASS}

It is another new emerging Radio based satellite navigation system. Initially the GLONASS was developed in 1976 for the use of military, to overcome the problems of Tsikada system. Tsikada system was able to provide accurate position but the processing time was 1 to $2 \mathrm{Hrs}$ for signal processing [5]. In this way till twenty six satellites were obtained in orbit by 1995 , but due to failures and satellite design expiry older satellites were taken away from the project. Hence, till 2001 only eight satellites there were presrent in GLONASS. As the Russian Government was an invested lot of fund in this project, to change this situation. 


\section{International Journal of Science and Research (IJSR) \\ ISSN (Online): 2319-7064}

Index Copernicus Value (2013): 6.14 | Impact Factor (2014): 5.611

The Russian government organized a program. Global Navigation System on $20^{\text {th }}$ August 2001[5]. The Indian government also joined this program and become a partner of GLONASS to ensure funding. On May 18,2007 Russian president Vladimir Patin' signed a decree(wwwGLON), to provide open access to civilian navigation signals of the GLONASS system to Russian and foreign consumers for free of charge and without any limitations. As the development and maintenance of GLONASS system is conducted by Petrol space agency (ROSCOSMOS, MOD). In 2010 GLONASS had achieved $100 \%$ coverage of Russia's territory and in Oct 2011 the full orbital constellation of 24 satellites was restored, giving full global coverage.

\section{Features:}

- Satellite Constellation: 24 Satellites (21 Active and 3 Redundant)

- Multiple Access: FDMA

- Modulation Schemes used: BPSK(5.11), BPSK(4)

- Center Frequency: 1598.0625 MHz-1605.375 MHz

- Frequency Bands: L1, L2 and L3 [7].

\section{GALILEO}

Galileo, the first satellite, navigation, timing and positing system specifically designed for applications in civil, will offer state services with outstanding guarantee, integrity, availability and accuracy. [6]. It is a joint initiative of the European Commission. It was completed in 2003, produced the basic specifications for the required system. It will be validated by deploying four satellites of the overall constellation together with the control centre and ground stations. Four satellites are the minimum to guarantee precise positioning and time services at specific locations [6]. Early in this phase, the Galileo In-Orbit Validation Element mission will employ two satellites, GIOVE-A and GIOVE-B, and their mission- and ground-control. GIOVE is securing access to the Galileo frequencies allocated by the International Telecommunications Union, characterizing the radiation environment of the Medium Earth Orbits planned for the Galileo satellites, testing the most critical technologies that are like atomic clocks on board, signal generator and user receivers and characterizing the novel features of the Galileo signal design [8]. Galileo will begin full deployment, covering the entire ground network and launching the remaining 26 satellites to complete the constellation .

\section{Features:}

- Satellite Constellation: 30 Satellite (27 active +3 redundant).

- Multiple Accesses: CDMA.

- Modulation Scheme : CBOC,BOC, BPSK.

- Center Frequency: 1575.42 MHz, 1278.75 MHz, $1191.795 \mathrm{MHz}$

- Frequency Bands: E1, E6 and E5.

According to survey GLONASS \& GALILEO provides more accurate results than GPS but their receiver costs very high. As per our the project is concern, GPS is the best technology considering its availability and receiver cost. Because today everyone is using Android phone that comes with inbuilt GPS receiver installed in it. Therefore there is no need of purchasing a separate GPS receiver for each client.

\section{DGPS}

Differential GPS is recent technology that has two antennas placed in module each. Both antennas gives differential value of the longitude and latitude. Depending upon the differential value correct location can be traced. DGPS more advantages than GPS. It has more accurate value. Range is also greater than GPS. That's why we are using DGPS in our project.

\section{Proposed Methodology}

\section{Project Components}

\section{1) Sensors}

We are using different types of sensors for safety of travelers like carbon monoxide sensor, temperature sensor, and for theft control we are using fuel level sensor. All the data from sensors has been stored in embedded board and sent to owner of the vehicle via GSM GPRS module.

\section{2) Transmitting Unit}

Transmitting Side contains GPS, GSM and GPRS functionality which is preloaded in a vehicle.

a) GPS:

The United States Department of Defense (DoD) has developed GPS, which is an all-weather, space based navigation system to meet the needs of the USA military forces. GPS has made a impact on almost all positioning, navigation, timing and monitoring applications.

b) Advance GSM:

A GSM modem is a modem which is wireless that works with a GSM wireless network. It behaves like a Dial-up modem. The working of GSM modem is based on commands. GSM Provides recommendation not the Requirement. Advance GSM provides better accuracy and hence results. It defines functions and interfaces requirement in detail but do not address the hardware.

c) Receiving or monitoring unit

Monitoring unit can be an Android Application or a Web Application through which user will get to know the actual position of proposed vehicle. The Application will display the different co-ordinates i.e. longitude and latitude receiver from tracking device and plot them on Google Maps.

\section{Work Flow}

\section{Tracking Device}

1) The tracking device will continuously request to the GPS satellite for its location information.

2) At the same time GPS satellite will provide the location information to tracking device installed in vehicle.

3) The tracking device will send the location information back to the mobile or server through GPRS and continuously update the database. 


\section{Monitoring Device}

1) Monitoring device will continuously monitor the database from server.

2) From that database the location information will be plotted on Google maps.

\section{Applications}

Departments like Police Department, Military. To keep track of city Buses, State Transport Buses, Public Buses, Private Buses and as well as Vehicles. Companies like Food Delivery, Car Rental and College Transport can also use this system.

\section{Conclusion}

This proposed system allows organizations to track their vehicles and to get exact location of vehicle. The system allows those companies to monitor the travelled routes through a web client that uses the Google Map and shows colors on the map to indicate if the devices on route. The general result is that the system proved to be reliable as to view the positioning of the devices.

\section{Acknowledgment}

We would like to take this opportunity to express our gratitude and deep regard to our project guide - Prof. H. K. Bhangale, for his guidance, valuable feedback and for constant encouragement for the project. Working under him was extremely knowledgeable experience for us.

\section{References}

[1] Abid Khan \& Ravi Mishra, -GPS - GSM Based Tracking Systemll, International Journal of Trends and Technology, ISSN: 2231 - 5381, Volume 3, Issue 2, 2012

[2] Rodrigo R. Oliveira, Felipe C. Noguez, Cristiano A. Costa, Jorge L. Barbosa \& Mario P. Pardo, -SWTRACK: An Intelligent Model for Cargo Tracking based on off-the-shelf Mobile Devicesll, ELSEVIER Expert Systems with Applications 40 (2013) 2023 - 2031

[3] Global Positioning System Standard Positioning Service Performance Standardll, Department of Defence - USA, 4th Edition (2008) $\backslash$

[4] Zechun Huang, Dingfa Huang, Zhu Xu \& Zhigen Xu, -GPS Vehicle Positioning Monitoring System Integrated with CORS and Mobile GISI, ELSEVIER Procedia Environmental Sciences 10(2011)2498-2504

[5] Federal Space Agencyll - Information Analytical Centre [https://glonass-iac.ru/en/index.php]

[6] Andrew Wilson, - The First Galileo Satellites GIOVEll, ESA Publications Division (BR-251)

[7] Tushar Saxena, Deepak Kumar, J.S. Jadon, -A Literature Study of Various Satellite Navigation Systems with Reference to Their Signalling Schemell, International Journal of Research Aspects of Engineering and Management, ISSN: 2348-6627, Vol. 1, Issue 1, FEB 2014 